\title{
A Case of Hereditary Spherocytosis Initially Manifested as an Aplastic Crisis Caused by Parvovirus B19 Infection
}

\author{
Hyungsuk Jin ${ }^{1}$, Ji Won Lee ${ }^{1}$, Hee Young Ju', Hee Won Cho', Ju Kyung Hyun', \\ Ki Woong Sung ${ }^{1}$, Hong Hoe Koo', Hee-Jin $\mathrm{Kim}^{2}$ and Keon Hee Yoo ${ }^{1}$ \\ ${ }^{1}$ Department of Pediatrics, Samsung Medical Center, Sungkyunkwan University School of Medicine, \\ ${ }^{2}$ Department of Laboratory Medicine and Genetics, Samsung Medical Center, Sungkyunkwan University School of Medicine, Seoul, Korea
}

Hereditary spherocytosis (HS) is the most common inherited red cell membrane disorder. Its main laboratory finding is anemia with reticulocytosis. However, in the case of an aplastic crisis, there may be no reticulocytosis, making the diagnosis of HS difficult. We present the case of a 4-year-old boy who initially presented with persistent fever and sore throat. His 8-year old brother also had anemia of unknown etiology, and his father had a history of splenectomy in his 20s. Physical examination revealed anemic conjunctivae and hepatosplenomegaly, and laboratory findings showed anemia with decreased reticulocyte count and elevated ferritin and lactate dehydrogenase levels. A peripheral blood smear showed microcytic hypochromic anemia with severe poikilocytosis (spherocytes, acanthocytes, schistocytes), and bone marrow examination revealed decreased erythroid cells and increased hemophagocytosis. Increased osmotic fragility was observed, and parvovirus B19 was detected using polymerase chain reaction. Hence, we established the diagnosis of hereditary spherocytosis manifested as an aplastic crisis caused by parvovirus B19 infection.

Key Words: Aplastic crisis, Parvovirus B-19, Hereditary spherocytosis
pISSN 2233-5250 / elSSN 2233-4580 https://doi.org/10.15264/cpho.2020.27.2.120 Clin Pediatr Hematol Oncol 2020;27:120 123

Received on July 1, 2020

Revised on August 18, 2020

Accepted on September 19, 2020

Corresponding Author: Keon Hee Yoo Department of Pediatrics, Samsung Medical Center, Sungkyunkwan University School of Medicine, 81 Irwon-ro, Gangnam-gu, Seoul 06351, Korea

Tel: $+82-2-3410-3532$

Fax: $+82-2-3410-0043$

E-mail: keonhee.yoo@samsung.com ORCID ID: orcid.org/0000-0002-5980-7912

\section{Introduction}

Hereditary spherocytosis (HS) is the most common inherited red cell membrane disorder, and affects 1 in 2,000-3,000 individuals [1]. It is inherited mostly in an autosomal dominant pattern [2]. HS is caused by a deficiency in a specific protein, which is part of the red blood cell (RBC) membrane cytoskeleton, eventually shaping the RBC as a spherocyte. As the spherocyte is easily destroyed by macrophages in the spleen, anemia, jaundice, and splenomegaly develop as common symptoms. In addition, $\mathrm{RBC}$ production increases and reticulocytes are usually increased [3].

Parvovirus B19 is a cause of viral exanthem of in- fectious origin and is one of the causes of acute arthritis in adults. In healthy hosts, Parvovirus B19 generally causes self-limiting subclinical erythroid aplasia, followed by rash or arthralgia. However, in patients with diminished production or increased loss of erythrocytes, Parvovirus B19 infection can result in a severe drop in hemoglobin levels, which could be life-threatening and is called 'aplastic crisis' [4].

Here we report a case of HS which was initially presented as an aplastic crisis caused by Parvovirus B19 infection.

\section{Case Report}

A 4-year-old boy was transferred to our hospital due 
to persistent anemia. He developed fever up to $40.1^{\circ} \mathrm{C}$ and sore throat 2 weeks before. He was diagnosed with acute pharyngotonsillitis at a local clinic and prescribed antibiotics. Because of persistent fever for more than 1 week, he was transferred to another hospital, where intravenous antibiotics (cefotaxime and azithromycin) were administered. Methylprednisolone (1 mg/kg/dose bid) was administered from day 4 after admission due to persistent fever, and fever subsided. On admission, the patient had microcytic hypochromic anemia (hemoglobin, $7.3 \mathrm{~g} / \mathrm{dL} ; \mathrm{MCV}, 70.6 \mathrm{fL} ; \mathrm{MCH}, 26.5 \mathrm{~g} / \mathrm{dL}$; reticulocyte, $5.8 \%$ ), and the hemoglobin level decreased to $4.0 \mathrm{~g} / \mathrm{dL}$ He was transferred to our hospital with the impression of hemophagocytic lymphohistiocytosis (HLH).

He was born at full-term through a cesarean section (due to a previous cesarean section). No past medical history was noted to date. Family history revealed an 8year old brother with anemia of unknown etiology and a father, who had had a splenectomy in his 20s. Upon arrival at our hospital, the fever and sore throat had subsided. On physical examination, anemic conjunctivae and hepatosplenomegaly (liver 4 fingerbreadth and spleen 1 fingerbreadth) were observed.

Laboratory findings were as follows: white blood cell $7,290 \times 10^{3} / \mu \mathrm{L}$; hemoglobin $4.8 \mathrm{~g} / \mathrm{dL}$; mean corpuscular volume $15.0 \mathrm{fL}$, mean corpuscular hemoglobin (MCH) $23.3 \mathrm{~g} / \mathrm{dL}$, mean corpuscular hemoglobin concentration (MCHC) $32.0 \mathrm{~g} / \mathrm{dL}$, reticulocyte $0.30 \%$, platelet count $235 \times 10^{3} / \mu \mathrm{L}$, absolute neutrophil count $3,650 \times 10^{3} / \mu \mathrm{L}$ aspartate aminotransferase (AST) $41 \mathrm{IU} / \mathrm{L}$, alanine aminotransferase (ALT) $31 \mathrm{IU} / \mathrm{L}$, total bilirubin $0.8 \mathrm{mg} / \mathrm{dL}$, ferritin $1,599.2 \mu \mathrm{g} / \mathrm{L}(15-200 \mu \mathrm{g} / \mathrm{L})$, haptoglobin $0.35 \mu \mathrm{mol} / \mathrm{L}$
(9.76-31.4 $\mu \mathrm{mol} / \mathrm{L})$, triglyceride $2.29 \mathrm{mmol} / \mathrm{L}$ (0.68-1.69 $\mathrm{mmol} / \mathrm{L}$ ), and lactate dehydrogenase (LD) $8.65 \mathrm{U} / \mathrm{L}$ (1.67$3.17 \mathrm{U} / \mathrm{L})$. Direct and indirect Coombs tests were negative, and peripheral blood smears showed microcytic hypochromic anemia with severe poikilocytosis (spherocytes, acanthocytes, schistocytes) (Fig. 1A). Bone marrow examination was performed to exclude the possibility of hematologic malignancy, and it showed decreased erythroid cells (Fig. 1B) and increased hemophagocytosis (Fig. 1C). Considering his family history, hereditary spherocytosis was the most likely diagnosis, and the osmotic fragility test showed an increased osmotic fragility (Fig. 2). Parvovirus B19 was detected by polymerase chain reaction (PCR), and the titer of parvovirus B19 in whole blood was 9,938 IU/ $\mu \mathrm{L}$. We established a diagnosis of hereditary spherocytosis manifested as an aplastic crisis caused by parvovirus B19 infection.

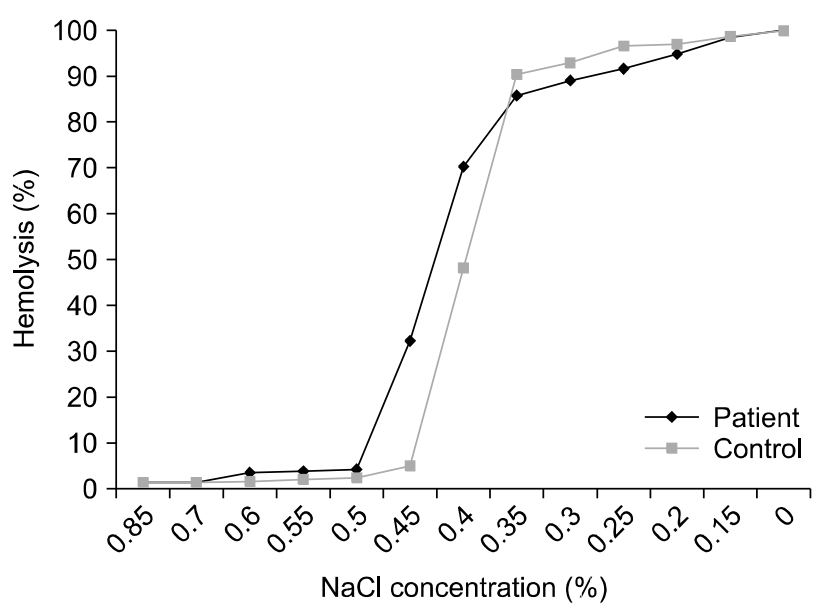

Fig. 2. Osmotic fragility test. The osmotic fragility test showed an increased osmotic fragility.
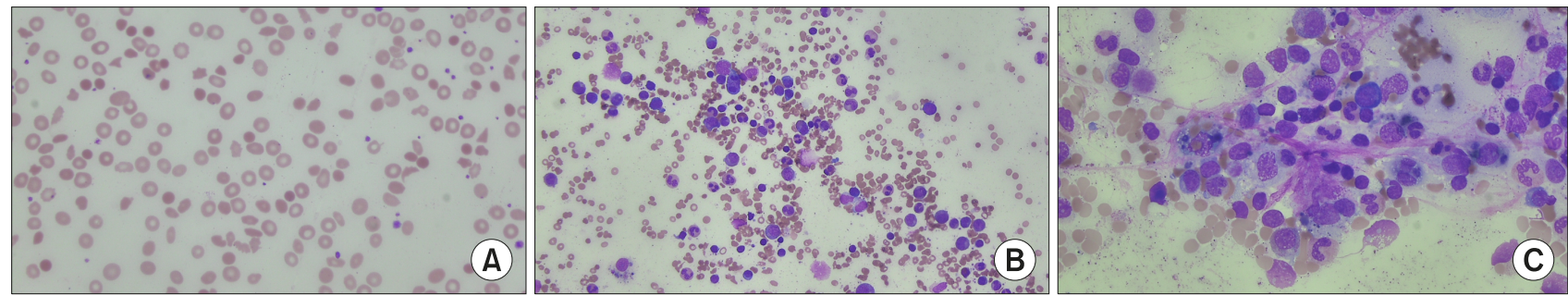

Fig. 1. Peripheral blood smears and bone marrow aspiration. Peripheral blood smears showed microcytic hypochromic anemia with severe poikilocytosis (spherocytes, acanthocytes, schistocytes) (A). Bone marrow examination showed decreased erythroid cells (B) and increased hemophagocytosis (C). 
During admission, red blood cell transfusion was performed, and hemoglobin levels increased gradually with reticulocyte recovery. He is currently undergoing regular follow-up with folic acid.

\section{Discussion}

Red cell membrane disorders are inherited diseases due to mutations in various membrane or skeletal proteins, resulting in decreased red cell deformability, reduced life span, and premature removal of the erythrocytes from circulation [5]. One of the important components of the red blood cell membrane is the cytoskeleton, such as spectrin $\alpha$ and $\beta$-chains, proteins 4.1 , or $4.1 \mathrm{R}$, and actin, and HS is caused by defects that interrupt the spectrin-actin interaction [6]. The typical clinical symptoms of HS are pallor due to anemia, jaundice due to hyperbilirubinemia and splenomegaly, but HS shows a broad spectrum of clinical severity, ranging from asymptomatic to severe transfusion-dependent forms. Asymptomatic or mildly anemic patients are often diagnosed after aplastic crises [6]. Reticulocytosis is the main laboratory finding of HS, but there may not be an increase of reticulocytes in aplastic crisis, as in this case, making the diagnosis difficult.

Infection may cause a transient aplastic crisis in patients with increased red cell destruction, and $70-80 \%$ of aplastic episodes in predisposed individuals are caused by parvovirus B19 infection [4]. Although erythrocytes are predominantly affected, concurrent thrombocytopenia, neutropenia, or pancytopenia are found infrequently, and aplasia may also be associated with a hemophagocytic syndrome [7-9]. While anemia may be lethal, the aplastic crisis itself usually ends with the appearance of specific antibodies and rarely lasts for more than 2 weeks [4].

In this case, HS was diagnosed based on spherocytes in the peripheral blood smear and increased osmotic fragility. Family history (history of splenectomy in father and anemia of unknown etiology in brother) also supported the diagnosis. Interestingly, this patient was initially transferred to our hospital with the impression of
HLH because of fever, anemia and elevated ferritin level. Hemophagocytosis was also observed in the bone marrow aspiration. Rarely, parvovirus B19 is known to cause HLH [4], and parvovirus B19-induced HLH has also been reported in HS patients [10]. Therefore, if a patient is suspected to have HLH, aplastic crisis should be considered as a differential diagnosis, and vice versa.

The diagnosis of HS is based on clinical features, positive family history, and the observation of a peripheral blood smear. Patients with a family history of HS and typical HS biological manifestations do not require any additional tests according to the 2011 guidelines revised by the General Haematology Task Force of the British Committee for Standards in Haematology [3]. However, currently, according to the updated standard operating procedure for the diagnosis of hereditary hemolytic anemia (HHA) made by RBC Disorder Working party of the Korean Society of Pediatric Hematology-Oncology, more specific biological test may be required when HHA with membranopathy is suspected [11]. The osmotic fragility test is a traditional method that uses the property of spherocytes, which easily hemolyze in hypoosmotic solutions due to their surface-to-volume area, but its sensitivity for diagnosis is low and can give false negative results in $10-20 \%$ of HS cases and false positive results in autoimmune hemolytic anemia [11,12]. Recently, the Eosin 5-malemide (EMA) binding test using flow cytometry was introduced in the diagnosis of HS with high sensitivity and specificity. It measures the mean red cell fluorescence following labeling with the EMA dye to document surface area loss [1]. Analysis of major erythrocyte membrane proteins via sodium dodecyl sulfate-polyacrylamide gel electrophoresis (SDS-PAGE) or next-generation sequencing test to detect causative gene variants can also be used for diagnosis [6].

Other than Parvovirus B19 virus, other viral or bacterial infections and megaloblastic crisis caused by folate deficiency also can cause hypoplastic crisis. The clinical manifestations of HS vary widely. The frequency of progression to aplastic crisis in HS patients has not been clearly reported. In a retrospective study of $63 \mathrm{HS}$ pediatric patients, 3 patients (4.8\%) developed an aplastic cri- 
sis [13]. During these crisis, high RBC turnover in the setting of erythroid marrow failure can result in profound anemia, high output heart failure, cardiovascular collapse. Leukocyte and platelet count may also fall. Considering the various causes and manifestations of aplastic crisis will be helpful in the diagnosis of anemia of unknown cause.

In conclusion, we report a case of HS initially presented as an aplastic crisis caused by Parvovirus B19 infection. Reticulocytosis is the main laboratory finding of HS, but the possibility of an aplastic crisis should also be considered during the diagnostic work-up of anemia with decreased reticulotye counts. Furthermore, we have to remember that detailed history taking, including family history, is always important.

\section{Conflict of Interest Statement}

The authors have no conflict of interest to declare.

\section{References}

1. Da Costa L, Galimand J, Fenneteau O, Mohandas N. Hereditary spherocytosis, elliptocytosis, and other red cell membrane disorders. Blood Rev 2013;27:167-78.

2. Shah S, Vega R. Hereditary spherocytosis. Pediatr Rev 2004; 25:168-72.
3. Bolton-Maggs PH, Langer JC, Iolascon A, Tittensor P, King MJ; General Haematology Task Force of the British Committee for Standards in Haematology. Guidelines for the diagnosis and management of hereditary spherocytosis--2011 update. Br J Haematol 2012;156:37-49.

4. Heegaard ED, Brown KE. Human parvovirus B19. Clin Microbiol Rev 2002;15:485-505.

5. Barcellini W, Bianchi P, Fermo E, et al. Hereditary red cell membrane defects: diagnostic and clinical aspects. Blood Transfus 2011;9:274-7.

6. Andolfo I, Russo R, Gambale A, Iolascon A. New insights on hereditary erythrocyte membrane defects. Haematologica 2016; 101:1284-94

7. Saarinen UM, Chorba TL, Tattersall P, et al. Human parvovirus B19-induced epidemic acute red cell aplasia in patients with hereditary hemolytic anemia. Blood 1986;67:1411-7.

8. Lefrère JJ, Couroucé AM, Bertrand Y, Girot R, Soulier JP. Human parvovirus and aplastic crisis in chronic hemolytic anemias: a study of 24 observations. Am J Hematol 1986;23:271-5.

9. Sato T, Ueda D, Sakota S, Haseyama K, Chiba S, Kudoh T. Pancytopenia with hemophagocytosis secondary to parvovirus B19 infection in a family with hereditary spherocytosis. Pediatr Int 1999;41:561-4.

10. Tanaka Y, Takahashi Y, Kimura Y, et al. Human parvovirus B19-induced hemophagocytic lymphohistiocytosis and myocarditis in an adult patient with hereditary spherocytosis. Rinsho Ketsueki 2018;59:682-7.

11. Jung HL. A new paradigm in the diagnosis of hereditary hemolytic anemia. Blood Res 2013;48:237-9.

12. Ciepiela O. Old and new insights into the diagnosis of hereditary spherocytosis. Ann Transl Med 2018;6:339.

13. Oliveira MC, Fernandes RA, Rodrigues CL, Ribeiro DA, Giovanardi MF, Viana MB. Clinical course of 63 children with hereditary spherocytosis: a retrospective study. Rev Bras Hematol Hemoter 2012;34:9-13. 